\title{
Postoperative sepsis after colorectal surgery: a prospective single-center observational study and review of the literature
}

\author{
Francesk Mulita ${ }^{1}$, Elias Liolis ${ }^{2}$, Karolina Akinosoglou ${ }^{3}$, Levan Tchabashvili ${ }^{1}$, Ioannis Maroulis ${ }^{1}$, \\ Charalampos Kaplanis ${ }^{1}$, Michail Vailas ${ }^{1}$, George Panos ${ }^{3}$ \\ ${ }^{1}$ Department of General Surgery, General University Hospital, Patras, Greece \\ 2Department of Internal Medicine, Division of Oncology, General University Hospital, Patras, Greece \\ ${ }^{3}$ Department of Internal Medicine and Infectious Diseases, General University Hospital, Patras, Greece
}

Gastroenterology Rev 2022; 17 (1): 47-51

DOI: https://doi.org/10.5114/pg.2021.106083

Key words: sepsis, colorectal surgery, postoperative complication.

Address for correspondence: Dr. Francesk Mulita, Department of General Surgery, General University Hospital, Patras, Greece, e-mail: oknarfmulita@hotmail.com

\begin{abstract}
Introduction: Postoperative sepsis represents a significant problem in colorectal surgery patients.

Aim: To identify the etiology as well as the risk factors associated with the development of postoperative sepsis, based on prospective data of patients undergoing colorectal surgery at a single large-volume institution.

Material and methods: Between November 2019 and February 2021, 141 patients underwent an elective operation for colorectal cancer at a tertiary hospital center. The following variables were recorded for each patient: age, gender, American Society of Anesthesiologists Classification (ASA class), duration of surgery, surgical approach, comorbidities (diabetes, cardiovascular disease, respiratory disease). Univariate analysis was performed using $\chi^{2}$ tests for categorical variables.

Results: A total of 69 males and 72 females were enrolled. Postoperative sepsis was diagnosed in 18 (12.77\%) cases, with anastomotic leakage being the most frequent cause (3.55\%). There was no statistically significant difference in the presence of sepsis among patients when gender, surgical approach, duration of surgery, and respiratory disease were taken into account. Sixty-nine patients were $>65$ years old, with sepsis being statistically significant in this group $(p=0.034)$. Furthermore, patients with ASA class $\leq 2$ developed postoperative sepsis less frequently than patients with advanced ASA scores $(p=0.008)$. Diabetes and cardiovascular disease also reach statistical significance; sepsis was more frequent in this group of patients $(p=0.013$ and $p=0.009$, respectively).

Conclusions: Following colorectal cancer procedures, postoperative sepsis was significantly more common among patients over 65 years old, ASA score > 2, and also with associated comorbidities such as diabetes and cardiovascular disease.
\end{abstract}

\section{Introduction}

Sepsis is a serious clinical syndrome that is associated with high morbidity and mortality rates for patients suffering from it. It is nowadays considered a major public health problem, as it is estimated that it costs more than $\$ 20$ billion annually for US health care systems [1, 2]. The number of sepsis cases per year also shows incremental growth lately, possibly due to the increase of the aging population. Sepsis is a clinical problem of great importance, which usually is treated with antibiotic medications and intravenous fluids. It should also be highlighted that very often people who manage to survive have long-term health problems and disabilities [2, 3].

Postoperative sepsis is described as a surgical complication, involving patients immediately after surgical operations, associated with any type of infection that can lead to sepsis, severe sepsis, or septic shock [4]. Postoperative sepsis is a major health problem as it is estimated that it occurs in more than $1 \%$ of elective procedures and in more than $4 \%$ of non-elective procedures. However, epidemiological data on postoperative sepsis are limited [5]. According to the literature, 50\% of all postoperative sepsis cases occur after gastrointestinal, cardiovascular, or thoracic surgical procedures. 
The mortality rate of patients with postoperative sepsis is estimated to be nearly $25 \%$. Risk factors associated with increased risk for postoperative sepsis include age, race, and male sex. Diseases such as diabetes, chronic liver disease, chronic renal disease, and metastatic neoplasms also increase the risk for postoperative sepsis. Reduction of its incidence and better outcomes rely on prophylaxis, recognition of sepsis at an early stage, and appropriate treatment [4].

Postoperative sepsis is also a major issue for patients with cancer, who are considered surgical candidates. According to a study, esophagectomy patients have the highest prevalence of sepsis, with the highest frequency of sepsis-related mortality being observed in lung resection patients. Needless to say, while the incidence of postoperative sepsis increases, associated mortality rates remain stable, possibly due to better hospital care and a decrease in disparities between hospitals [6]. Only a few studies have reported on risk factors for postoperative sepsis in colorectal surgery.

\section{Aim}

The aim of this study was to identify the risk factors and also the causes for developing sepsis based on a prospective analysis of patients undergoing colorectal surgery in a single center between November 2019 and February 2021.

\section{Material and methods}

This prospective study was conducted at the General University Hospital of Patras in Greece, an 800-bed tertiary Hospital in South-Western Greece that covers a population of approximately 1.5 million people, between November 2019 and February 2021, and included 141 patients undergoing elective operation for colorectal cancer.

Inclusion criteria were patients older than 18 years, elective surgery cases, preoperative hospital stay less than $48 \mathrm{~h}$, and diagnosis of colorectal cancer that was scheduled to be treated by elective colorectal surgery. Exclusion criteria were patients younger than 18 years old, emergency surgery, preoperative hospital stay more than $48 \mathrm{~h}$, and other non-malignant colorectal diseases such as infectious bowel disease, or diverticulitis. The patients gave informed written consent to the study. The day prior to surgery patients underwent preparation of the large bowel as well as antibiotic prophylaxis based on standards established at our institution: $2^{\text {nd }}$ generation cephalosporin and metronidazole administered $30 \mathrm{~min}$ before the first skin incision, and two doses of each antibiotic administered again after $4 \mathrm{~h}$ of operation.
All operations were conducted by the same group of surgeons and anesthesiologists. Patients received either a povidone-iodine antisepsis regimen, or chlorhexidine-alcohol skin preparation regimen.

The day before surgery, all patients provided written informed consent. Ethical approval was obtained from the Ethics Committee of the General University Hospital of Patras (No 5461/40626-11/11/2019).

After the patient's extubation in the operating room, surgical information was recorded (surgery time, intra-operative complications). Following surgery, patients were transferred to the surgical ward. The patient follow-up, data collection and analysis were carried out by our team of surgeons from the day of the surgery until hospital discharge.

The following variables were recorded for each patient: age ( $\leq 65$ or $>65$ years), gender (male/female), American Society of Anesthesiologists Classification (ASA class $\leq 2$ - healthy patients or with a mild systemic disease, or $>2$ - patients with at least one severe systemic disease) [7], duration of surgery ( $\leq 180 \mathrm{~min}$ or $>180 \mathrm{~min}$ ), surgical approach (open or laparoscopic), comorbidities (diabetes, cardiovascular disease, respiratory disease). The criteria used to define postoperative sepsis are those established by the Third International Consensus Definitions for Sepsis and Septic Shock (Sepsis-3) [3].

\section{Statistical analysis}

Univariate analysis was performed using the $\chi^{2}$ test for categorical variables to compare results between groups (SSI group vs non-SSI group). Values of $p<0.05$ were considered statistically significant.

\section{Results}

From November 2019 to February 2021 a total of 141 patients with colorectal cancer, including 69 males and 72 females, met the inclusion criteria for this prospective study. Postoperative sepsis was diagnosed in $18(12.77 \%)$ patients. In 5 (3.55\%) patients diagnosed with postoperative sepsis the etiology was anastomotic leakage, whereas the etiology of sepsis in 4 (2.84\%) patients was surgical site infection. Less common causes of postoperative sepsis were respiratory infection, central venous catheter-related infection, intra-abdominal abscess formation, urinary tract infection, pseudomembranous colitis, and postoperative ileus (Table I).

Univariate analysis showed no difference with regard to postoperative sepsis prevalence when patient gender, surgical approach, duration of the procedure, and respiratory disease were evaluated (Table II). Postoperative sepsis was significantly more frequent in the group of patients $>65$ years old compared to that of 
patients $\leq 65(18.84 \%$ vs. $6.94 \% ; p=0.034)$. There were $93(65.96 \%)$ patients with ASA class $\leq 2$, a group in which postoperative sepsis occurred significantly less frequently compared to those with ASA class $>2(7.53 \%$ vs. $22.92 \% ; p=0.009)$. In the present study, there were 20 (14.18\%) patients with diabetes and 19 (13.48\%) patients with cardiovascular disease. Postoperative sepsis occurred more often in these patients compared to patients without these two comorbidities (30\% vs. 9.92\%; $p=0.013$ and 31.58 vs. 9.84; $p=0.008$, respectively).

\section{Discussion}

The first definition of sepsis was developed at a 1991 consensus conference and sepsis was defined as a suspected infection leading to systemic inflammatory response syndrome (SIRS), which when accompanied by organ dysfunction was defined as severe sepsis. Severe sepsis could also lead to septic shock. An effort to update these definitions was made in 2001 by adding more diagnostic criteria but the definitions finally remained unchanged [1-3]. In 2016 the new definition of sepsis, called Sepsis-3, was announced. According

Table I. Etiology of postoperative sepsis in 141 patients undergoing colorectal surgery

\begin{tabular}{lc} 
Patient groups & $\boldsymbol{N}(\%)$ of patients \\
\hline Non-sepsis & $123(87.23)$ \\
\hline Sepsis: & $18(12.77)$ \\
\hline Anastomotic leakage & $5(3.55)$ \\
\hline Surgical site infection & $4(2.84)$ \\
\hline Respiratory infection & $2(1.42)$ \\
\hline Central venous catheter-related infection & $2(1.42)$ \\
\hline Intra-abdominal abscess formation & $2(1.42)$ \\
\hline Urinary tract infection & $1(0.71)$ \\
\hline Pseudomembrane colitis & $1(0.71)$ \\
\hline Ileus & $1(0.71)$
\end{tabular}

Table II. Univariate analysis of demographic and clinical characteristics of the patients

\begin{tabular}{|c|c|c|c|c|c|}
\hline \multirow[t]{2}{*}{ Variable } & \multicolumn{3}{|c|}{$N(\%)$ of patients in the group } & \multirow[t]{2}{*}{ Sepsis \% } & \multirow[t]{2}{*}{$P$-value } \\
\hline & Total $(n=141)$ & Sepsis $(n=18)$ & Non-sepsis $(n=123)$ & & \\
\hline Age: & & & & & 0.034 \\
\hline$\leq 65$ & $72(51.06)$ & $5(27.78)$ & $67(54.47)$ & 6.94 & \\
\hline$>65$ & $69(48.94)$ & $13(72.22)$ & $56(45.53)$ & 18.84 & \\
\hline Gender: & & & & & 0.548 \\
\hline Male & $69(48.94)$ & $10(55.56)$ & $59(47.97)$ & 14.49 & \\
\hline Female & $72(51.06)$ & $8(44.45)$ & $64(52.03)$ & 11.11 & \\
\hline ASA class: & & & & & 0.009 \\
\hline$\leq 2$ & $93(65.96)$ & $7(38.89)$ & $86(69.92)$ & 7.53 & \\
\hline$>2$ & $48(34.04)$ & $11(61.11)$ & 37 (30.08) & 22.92 & \\
\hline Duration of surgery [min]: & & & & & 0.375 \\
\hline$>180$ & $57(40.43)$ & $9(50.00)$ & $48(39.02)$ & 15.79 & \\
\hline$\leq 180$ & $84(59.57)$ & $9(50.00)$ & $75(60.98)$ & 10.71 & \\
\hline Surgical approach: & & & & & 0.574 \\
\hline Open & $119(84.40)$ & $16(88.89)$ & $103(83.74)$ & 13.45 & \\
\hline Laparoscopic & $22(15.60)$ & $2(11.11)$ & $20(16.26)$ & 9.09 & \\
\hline \multicolumn{6}{|l|}{ Comorbidities: } \\
\hline Diabetes & & & & & 0.013 \\
\hline Yes & $20(14.18)$ & $6(33.33)$ & $14(11.38)$ & 30.00 & \\
\hline No & $121(85.82)$ & $12(66.67)$ & $109(88.61)$ & 9.92 & \\
\hline Cardiovascular disease: & & & & & 0.008 \\
\hline Yes & $19(13.48)$ & $6(33.33)$ & $13(10.57)$ & 31.58 & \\
\hline No & $122(86.52)$ & $12(66.67)$ & $110(89.43)$ & 9.84 & \\
\hline Respiratory disease: & & & & & 0.471 \\
\hline Yes & $30(21.28)$ & $5(27.78)$ & $25(20.33)$ & 16.67 & \\
\hline No & $111(78.72)$ & $13(72.22)$ & 98 (79.67) & 11.71 & \\
\hline
\end{tabular}


to this, sepsis is "a life-threatening organ dysfunction caused by a dysregulated host response to infection". According to this definition, they have also proposed some diagnostic criteria that are going to be further analyzed in the next section. However, there is still some debate regarding the Sepsis- 3 definition as it shows inconsistencies [8].

According to the new definition of sepsis, organ dysfunction is defined as an increase of two points or more in the Sequential Organ Failure Assessment (SOFA) score consequent to the infection. It should also be noted that for patients with infections, an increase of two SOFA points gives an overall mortality rate of $10 \%$. Moreover, a new screening tool for use outside of ICU settings is qSOFA or "HAT". This tool is designed to identify patients with suspected infections who are more likely to have poor outcomes typical of sepsis. These patients should have at least two of the following criteria: hypotension, with systolic blood pressure $\leq 100 \mathrm{~mm} \mathrm{Hg}$, altered mental status, tachypnea with a respiratory rate of $22 / \mathrm{min}$ or greater. However, qSOFA is not considered a diagnostic criterion of sepsis but a screening tool [8].

After colorectal surgery, a life-threatening and extremely challenging complication is intra-abdominal sepsis. More specifically, colon surgery has been linked with the highest risk for causing surgical site infection (SSI) and development of sepsis. The causes of postoperative sepsis after colorectal surgery and its prevention have been studied before based on current medical literature. According to Weledji (2018), who conducted a literature review, postoperative sepsis is the result of a complex interaction between the patient, the surgeon, and the disease. More specifically, the most important cause was the presence of viable organisms in the site before wound closure. Additionally, the role of surgeons is considered very important, and their surgical technique or decision-making, such as bowel preparation, the use of antibiotics, and tension applied at the anastomosis, can affect postoperative sepsis [9]. Regarding disease factors, it has been observed that when the operation is emergent, fecal contamination can lead to sepsis and a high mortality rate. Finally, patient factors include the patient's age, malnutrition, health status and comorbidity, lifestyle, etc. Choudhuri and Uppal (2013) studied 103 patients who underwent gastrointestinal surgery and had an anastomotic leak postoperatively. Seventy-two of them developed septic shock. Malignancies, chronic obstructive pulmonary disease (COPD), liver disease, heart disease, and presence of bacteremia were associated with septic shock $(p<0.05)$, meaning that co-morbidity can affect sepsis occurrence [10]. In general, the number of studies re- garding the causes of postoperative sepsis after colorectal surgery is limited. Most of them study risk factors or prevention measures and not specifically the causes of sepsis.

According to the literature, colorectal surgery has been associated with high risk of postoperative infectious complications. Surgical site infections are one of the most common postoperative complications following colorectal procedures (1-40\%, depending on the procedure), with many negative consequences for patients, such as extended hospital stay, morbidity, readmission, and death $[11,12]$. Furthermore, postoperative infectious complications consist of pulmonary infection (10\%) and urinary infection (5\%). Postoperative sepsis is considered to be one of the most common causes of death for patients treated in the intensive care unit (ICU) [13]. Recent studies have shown that postoperative sepsis can lead to deep vein thrombosis (DVT) and a decreased survival rate $[14,15]$. Intake of synbiotics and probiotics can decrease postoperative sepsis up to $38 \%$, whereas preoperative low albumin levels are associated with increased prevalence of sepsis $[16,17]$.

In the present study anastomotic leakage was the most frequent factor linked to sepsis. Because of the severity of this complication, it is imperative to identify this condition and act as early as possible [18]. Anastomotic leakage is considered to be one of the most frequent complications following colorectal surgery that adds to rates of mortality and various morbidities [19]. The prevalence of this complication varies from $1.8 \%$ to $19.2 \%$ depending on operative risk factors such as contamination, changes in blood pressure, and blood loss [20, 21].

In our study, the prevalence of postoperative sepsis was $12.77 \%$ and the most frequent cause was anastomotic leakage $(3.55 \%)$. The outcomes of this prospective study suggest that there was no statistically significant difference in the presence of postoperative sepsis among patients depending on their gender, surgical approach (open vs. laparoscopic), duration of surgery, and respiratory disease. Interestingly enough, the major findings of this prospective study were that age $>65$ years, ASA score $>2$, and comorbidities such as diabetes and cardiovascular disease were associated with significantly higher incidence of postoperative sepsis.

Although this study has important findings, it has several limitations. One limitation of this study that should be considered is that we did not record data of patients undergoing emergency operations. Another limitation of our study is the small number of participants from a single center. In conclusion, our study highlights the need for conducting prospective random- 
ized controlled trials including large numbers of participants. This study is one of the few that analyzes the etiology as well as risk factors of postoperative sepsis in patients undergoing colorectal surgery.

\section{Conflict of interest}

The authors declare no conflict of interest.

\section{References}

1. Singer M, Deutschman CS, Seymour CW, et al. The Third International Consensus definitions for sepsis and septic shock (sepsis-3). JAMA 2016; 315: 801-10.

2. Taeb AM, Hooper MH, Marik PE. Sepsis: current definition, pathophysiology, diagnosis, and management. Nutr Clin Pract 2017; 32: 296-308.

3. Napolitano LM. Sepsis 2018: Definitions and Guideline Changes. Surg Infect 2018; 19: 117-25.

4. Fried E, Weissman C, Sprung C. Postoperative sepsis. Curr Opin Crit Care 2011; 17: 396-401.

5. Vogel TR, Dombrovskiy VY, Carson JL, et al. Postoperative sepsis in the United States. Ann Surg 2010; 252: 1065-71.

6. Sammon JD, Klett DE, Sood A, et al. Sepsis after major cancer surgery. J Surg Res 2015; 193: 788-94.

7. Mulita F, Karpetas G, Liolis E, et al. Comparison of analgesic efficacy of acetaminophen monotherapy versus acetaminophen combinations with either pethidine or parecoxib in patients undergoing laparoscopic cholecystectomy: a randomized prospective study. Med Glas 2021; 18: 27-32.

8. Sinha S, Ray B. Sepsis-3: how useful is the new definition? J Anaesthesiol Clin Pharmacol 2018; 34: 542-3.

9. Weledji EP. Is patient factor more important than surgeon-related factor in sepsis prevention in colorectal surgery? Int J Surg Open 2018; 12: 29-36.

10. Choudhuri AH, Uppal R. Predictors of septic shock following anastomotic leak after major gastrointestinal surgery: an audit from a tertiary care institute. Indian J Crit Care Med 2013; 17: 298-303.

11. Morikane K, Honda H, Yamagishi T, et al. Factors associated with surgical site infection in colorectal surgery: the Japan nosocomial infections surveillance. Infect Control Hosp Epidemiol 2014; 35: 660-6.

12. Banaszkiewicz Z, Cierzniakowska K, Tojek K, et al. Surgical site infection among patients after colorectal cancer surgery. Pol Przegl Chir 2017; 89: 9-15.

13. Rovera F, Dionigi G, Boni L, et al. Infectious complications in colorectal surgery. Surg Oncol 2007; 16 Suppl 1: S121-4.

14. Hatch Q, Nelson D, Martin M, et al. Can sepsis predict deep venous thrombosis in colorectal surgery? Am J Surg 2016; 211: 53-8.

15. Aquina CT, Blumberg N, Becerra AZ, et al. Association among blood transfusion, sepsis, and decreased long-term survival after colon cancer resection. Ann Surg 2017; 266: 311-7.

16. Arumugam S, Lau CS, Chamberlain RS. Probiotics and synbiotics decrease postoperative sepsis in elective gastrointestinal surgical patients: a meta-analysis. J Gastrointest Surg 2016; 20: 1123-31.
17. Truong A, Hanna MH, Moghadamyeghaneh Z, Stamos MJ. Implications of preoperative hypoalbuminemia in colorectal surgery. World J Gastrointest Surg 2016; 8: 353-62.

18. Kirchhoff P, Clavien PA, Hahnloser D. Complications in colorectal surgery: risk factors and preventive strategies. Patient Saf Surg 2010; 4: 5.

19. Pak H, Maghsoudi LH, Soltanian A, Gholami F. Surgical complications in colorectal cancer patients. Ann Med Surg 2020; 55: 13-8.

20. van Rooijen SJ, Huisman D, Stuijvenberg M, et al. Intraoperative modifiable risk factors of colorectal anastomotic leakage: Why surgeons and anesthesiologists should act together. Int J Surg 2016; 36: 183-200.

21. Vallance A, Wexner S, Berho M, et al. A collaborative review of the current concepts and challenges of anastomotic leaks in colorectal surgery. Colorectal Dis 2017; 19: 01-12.

Received: 9.03.2021

Accepted: 12.04 .2021 